\title{
A Compactness Criterion of Mixed Krasnoselskiř-Riesz Type in Regular Ideal Spaces of Vector Functions
}

\author{
M. Väth
}

\begin{abstract}
We present a compactness criterion in ideal spaces of vector-valued functions. In the case of real functions, the criterion gives a precise formula for the measure of non-compactness. In the Lebesgue-Bochner spaces $L_{p}\left(\mathbb{R}^{n}, U\right)$ the result can be interpreted as a Riesz compactness criterion and generalizes a theorem of Orlicz and Szufla.
\end{abstract}

Keywords: Riesz compactness criterion, Krasnoselskii compactness criterion, ideal spaces, Köthe spaces, Banach function spaces, spaces of measurable functions, vectorvalued functions

AMS subject classiflcation: Primary 46 E 30, 46 E 40, secondary 45 N 10, 46 G 10

\section{The main result}

Let $S$ be a measure space and $(U,|\cdot|)$ be a Banach space. We call a function $x: S \rightarrow U$ (strongly Bochner) measurable if, on each set of finite measure, $x$ may be approximated a.e. (in the sense of the Lebesgue extension of the measure space) by a sequence of simple functions. This is the definition used, e.g., in [9]. For a measurable set $E \subseteq S$ we define the projection $P_{E}$ by $P_{E} x(s)=\chi E(s) x(s)$.

A normed linear space $(X,\|\cdot\|)$ of (classes of) measurable functions $x: S \rightarrow U$ is called a preideal space, if the relations $x \in X$ and $|y(s)| \leq|x(s)|$ for a measurable function $y$ imply that $y \in X$ and $\|y\| \leq\|x\|$. If $X$ is complete, it is called an ideal space. In some literature, preideal spaces are called (normed) Köthe spaces, and ideal spaces are called Banach function spaces; but sometimes additional requirements are imposed on these spaces. The proofs of properties for such spaces which are not given in this paper can be found in $[27,30,31]$.

To each preideal space $X$. one associates a preideal space $X_{\mathbb{R}}$ of real functions $x$ : $S \rightarrow \mathbb{R}$ in the obvious way by the relation

$$
x \in X \quad \Longleftrightarrow \quad|x| \in X_{\mathbb{R}} .
$$

M. Väth: University of Würzburg, Department of Mathematics, Am Hubland, D-97074 Würzburg; e-mail: vaeth@cip.mathematik.uni-wuerzburg.de

The paper was written while the author was visiting Caltech as a DAAD fellow. Financial support by the DAAD is gratefully acknowledged. The author thanks W. A. J. Luxemburg for stimulating discussions and valuable remarks on the subject. 
$X$ is an ideal space if and only if $X_{\mathbb{R}}$ is an ideal space. We will often not distinguish between $X$ and $X_{\mathbb{R}}$ and thus write, e.g., $\chi_{E} \in X$. We say that a family $M \subseteq X$ has equicontinuous norm in $X$, if

$$
\inf _{\operatorname{mes} E<\infty} \sup _{x \in M}\left\|P_{E} x\right\|=0 \quad \text { and } \quad \lim _{\delta \rightarrow 0} \sup _{\text {mes } D \leq \delta} \sup _{x \in M}\left\|P_{D} x\right\|=0 .
$$

The space $X$ is called regular, if all singletons $M=\{x\}$ with $x \in X$ have equicontinuous norm. For example, the Lebesgue-Bochner spaces $X=L_{p}(S, U)(1 \leq p \leq \infty)$ are regular if $p<\infty$. It will be convenient to introduce the shortcut

$$
M(s)=\{x(s): x \in M\}
$$

for a set $M \subseteq X$.

We are interested in estimating the Hausdorff measure of non-compactness of the set $M$ in relation to the Hausdorff measure of non-compactness of the sets $M(s)$. Such estimates play an important role in the study of integral and functional equations of vector-valued functions (see, e.g., $[20,21,23,26]$ ). Recall that the Hausdorff measure of non-compactness of a set $A$ in a metric space $Y$ is defined as the infimum of all $\varepsilon>0$ such that $A$ admits a finite $\varepsilon$-net in $Y$.

We denote the Hausdorff measure of non-compactness by $\chi(A)$ (or by $\chi Y(A)$, to emphasize the dependence on $Y$ ). Observe that $\chi_{Y}(A)$ increases, in general, if we replace $Y$ by a subspace $Y_{0} \subseteq Y$ with $A \subseteq Y_{0}$. Thus, it is not surprising that in order to get "good" estimates one has to impose geometric conditions on the space (in our case, on $U$ ). Indeed, for an analogous result in the space of continuous functions $[4,19]$ one looses the factor 2 if the space $U$ does not have certain geometric properties (the factor 2 can not be decreased by the examples in [12]). The geometric property which turned out useful for that case, is the following

Definition 1. We say that a Banach space $U$ has the $L$-retraction property, if for each separable subspace $U_{0} \subseteq U$ there exists a function $R: U \rightarrow U$ with the following properties:

1. The range of $R$ is separable, and $R(u)=u$ on $U_{0}$.

2. $R$ satisfies a Lipschitz condition with constant $L$.

If $L=1$, we say that $U$ has the retraction property.

We shall also need another geometric property:

Definition 2. We say that a Banach space $U$ has the $(p, q)$-exhaustion property, if each separable subspace $U_{0} \subseteq U$ is contained in a separable subspace $V \subseteq U$ with the following properties: There are bounded linear projections $P_{k}: V \rightarrow V$ and numbers $q_{k}$ such that:

1. The range $U_{k}=P_{k}(V)$ is a finite-dimensional subspace, where $U_{1} \subseteq U_{2} \ldots$ and $V=\overline{U U_{k}}$.

2. We have limsup $\left\|P_{k}\right\| \leq p$, limsup $q_{k} \leq q$, and

$$
\left|u-P_{k} u\right| \leq q_{k} \operatorname{dist}\left(u, U_{k}\right) \quad\left(u \in U_{0}\right)
$$


If $p=q=1$, we say that $U$ has the exhaustion property.

For applications, it is usually sufficient to put $q_{k}=\left\|I-P_{k}\right\|$ : For this choice, estimate (1) is always satisfied, even for all $u \in V$. Indeed, for any $u_{k} \in U_{k}$ we have $\left|u-P_{k} u\right|=\left|\left(I-P_{k}\right)\left(u-u_{k}\right)\right| \leq\left\|I-P_{k}\right\|\left|u-u_{k}\right|$.

Let us give some examples. Recall that a Banach space $U$ is called weakly compactly generated, if there is some weakly compact set $K \subseteq U$ whose linear span is dense in $U$. All separable spaces and all reflexive spaces are weakly compactly generated.

Example 1. Each separable Banach space $U$ has the retraction property. More general, if one assumes the axiom of choice, each weakly compactly generated Banach space has the retraction property. Indeed, if $U$ is weakly compactly generated, the existence of a (linear!) projection $R$ onto a separable subspace containing $U_{0}$ is proved in [3] with the axiom of choice (see also [8: Chapter $5, \S 2 /$ Theorem 3]; for related results see [7]).

Example 2. Each Hilbert space has the retraction and the exhaustion property: One may choose $R$ as the projection onto the element of best approximation in $U_{0}$ which is the orthonormal projection onto $U_{0}$. Observe that in this way a countable form of the axiom of choice is sufficient (we shall assume the so-called axiom of dependent choices throughout).

Example 3. Each separable Banach space $U$ with a (Schauder) base has the retraction and the $(p, q)$-exhaustion property. Indeed, it is well-known that the canonical projections $P_{k}$ are bounded (uniformly, by the uniform boundedness principle); see, e.g., [24: Chapter I, §3] or [14: Chapter 1]. Hence, we may put $p=\lim \sup \left\|P_{k}\right\|$ and $q=\limsup \left\|I-P_{k}\right\|$. In particular, each Banach space with a monotone base (i.e. $\left\|P_{k}\right\| \equiv 1$ ) has the $(1,2)$-exhaustion property.

Example 4. Each space $U=L_{p}\left(S, \mathbb{R}^{n}\right)(1 \leq p<\infty)$ with the counting measure on $S$ (e.g. $U=l_{p}$ or $U=l_{p}(S)$ ) has the retraction and the exhaustion property. Indeed, if $U_{0} \subseteq U$ is separable, it has countable support $E$. Put $R=P_{E}$, and observe that the canonical projections $P_{k}$ for the canonical base of $V=P_{E} U$ satisfy $\left\|P_{k}\right\|=\left\|I-P_{k}\right\|=1$.

Example 5. Each finite-dimensional space has the retraction and the exhaustion property.

To formulate our results as general as possible, we recall two other measures of non-compactness:

The Kuratowski measure of non-compactness $\alpha$ of a set $A$ in a metric space is defined as the infimum of all $\varepsilon>0$ such that $A$ admits a covering of finitely many sets of diameter less than $\varepsilon$.

The inner Hausdorff measure of non-compactness $\chi_{i}$ of a set $A \cdot$ in a metric space is defined as the infimum of all $\varepsilon>0$ such that $A$ has a finite $\varepsilon$-net in $A$, i.e. $\chi_{i}(A)=$ $\chi_{A}(A)$.

The Kuratowski and the inner Hausdorff measures of non-compactness have the advantage that they do not depend on the underlying space (only on the metric). The following estimates hold:

$$
\chi(A) \leq \chi_{i}(A) \leq \alpha(A) \leq 2 \chi(A) .
$$


One should be aware that $\chi_{i}$ is (in contrast to $\chi$ and $\alpha$ ) not monotone, in general.

Our main results are the following sufficient compactness criteria (which, as we shall see, are even necessary if $U$ has finite dimension). For a subset $M \subseteq X$ of a preideal space, we define the expression

$$
\omega(M)=\inf _{\left(E_{k}\right)} \sup _{x \in M} \inf _{u_{1}, \ldots, u_{n} \in U}\left\|x-\sum_{k=1}^{n} u_{k} \chi E_{k}\right\|
$$

where the infimum is taken over all systems of finitely many pairwise disjoint sets $E_{1}, \ldots, E_{\mathrm{n}}$ with $\chi E_{\mathrm{k}} \in X$.

Theorem 1. Let $X$ be a regular preideal space of functions $x: S \rightarrow U$ with a Banach space $U$, and $M \subseteq X$ be bounded and have equicontinuous norm in $X$. Assume that there is a function $y \in X$ with

$$
\sup _{n} \chi_{i}\left(M_{0}(s) \cap K_{n}\right) \leq y(s) \text { a.e. } \quad\left(M_{0} \subseteq M \text { countable }\right)
$$

for some bounded Borel sets $K_{n} \supseteq\{u \in U:|u| \leq n\}$. If $M$ is countable, it suffices that (3) holds for $M_{0}=M$. If $U$ has the $L$-retraction property, we may replace (3) by

$$
\sup _{n} L_{\chi}\left(M_{0}(s) \cap K_{n}\right) \leq y(s) \text { a.e. } \quad\left(M_{0} \subseteq M \text { countable }\right) .
$$

Then we have the estimate

$$
\chi_{i}(M) \leq 2(\omega(M)+2\|y\|) .
$$

Moreover, if $M$ is separable (in $X$ ), we even have

$$
\alpha(M) \leq 2(\omega(M)+2\|y\|) .
$$

If $U$ has the $(p, q)$-exhaustion property, we have

$$
\chi_{i}(M) \leq 2(p \omega(M)+q\|y\|)
$$

and if additionally $M$ is separable, even

$$
\chi X(M) \leq p \omega(M)+q\|y\| .
$$

For the scalar case $U=\mathbb{R}$, Theorem 1 implies that a bounded set $M \subseteq X$ is precompact if it has absolutely continuous norm and satisfies $\omega(M)=0$. This is a special case of Krasnoselskir's compactness criterion in the spaces $L_{p}(S, \mathbb{R})$ (see, e.g., [13: Lemma 1.1]): A set $M \subseteq L_{p}(S, \mathbb{R})$ is precompact if and only if it is precompact in measure and has absolutely continuous norm. In this sense, the condition $\omega(M)=0$ means that $M$ is precompact in measure. We will make this more precise in Corollary 1 . On the other hand, the condition $\omega(M)=0$ may be interpreted as some "equicontinuity" condition (in the norm) for the set $M$ : This condition means that it is possible to approximate the functions in $M$ uniformly by "step functions" with fixed steps. Thus, one might suspect 
some connections between the condition $\omega(M)=0$ and the compactness criterion of Riesz: A set $M \subseteq L_{p}(\mathbb{R}, \mathbb{R})$ is precompact if and only if it is equicontinuous in the norm in the sense that

$$
\lim _{h \rightarrow 0} \sup _{x \in M}\|x(\cdot+h)-x(\cdot)\|_{p}=0 .
$$

This result was generalized for ideal spaces in [11]. The connection of (9) with $\omega(M)=0$ will become clear in Theorem 3.

Let us remark that the assumption that $X$ be only a preideal space is slightly "too general" in Theorem 1, since each regular preideal space is a dense subspace of a regular ideal space (at least, if the underlying measure space is $\sigma$-finite). To see this, observe that it suffices to prove that $X$ is a dense subspace of an ideal space $\tilde{X}$, because the regular part of $\tilde{X}$ is closed by [27: Theorem 3.3.2]. To verify that $X$ is a subspace of an ideal space, it suffices to consider $U=\mathbb{R}$ in view of [27: Theorem 3.2.1]. But for this case the claim has been proved in [17] (see also $[15,16])(X$ has the property $(A, 0)$ by the regularity).

In the space $X=L_{1}(S, U)$ we may even drop the conditions on the geometry of $U$ and weaken the separability assumption on $M$ for (8), if we modify (2):

Theorem 2. Let $X=L_{1}(S, U)$ with a Banach space $U$, and $M \subseteq X$ be bounded and have equicontinuous norm in $X$. Assume that there is a function $y \in X$ such that (3) holds. If $M$ is countable, it suffices that (3) holds for $M_{0}=M$; if $U$ has the L-retraction property, we may replace (3) by (4). Put

$$
\omega_{1}(M)=\inf _{\left(E_{k}\right)} \sup _{x \in M}\left\|x-\sum_{k=1}^{n}\left(\frac{1}{\operatorname{mes} E_{k}} \int_{E_{k}} x(s) d s\right) \chi E_{k}\right\|
$$

where the infimum is taken over all systems of finitely many pairwise disjoint sets $E_{1}, \ldots, E_{n}$ of positive finite measure. Then we have the estimate

$$
\chi x(M) \leq \omega_{1}(M)+2\|y\| .
$$

If either $M$ is separable (in $X$ ) or $U$ is separable, we even have the estimate

$$
\chi x(M) \leq \omega_{1}(M)+\|y\| .
$$

For Theorem 1 it is worth noting that, for a separable Banach space $U$, the set $M \subseteq X$ is usually separable in $X$, because $X$ itself is separable: We call a measure space $S$ separable, if the system of measurable sets of finite measure with the metric $d(A, B)=\operatorname{mes}(A \Delta B)$ is separable. In particular, $S=\mathbb{R}^{n}$ is separable by this definition. This is the definition used in [30]. It can be proved that each regular preideal space $X$ of functions $x: S \rightarrow U$ is separable if $S$ and $U$ both are separable. This fact is not evident, but we skip the proof.

From this point of view, it is not so surprising that we already get a better estimate in Theorem 2 if $U$ is separable. However, the question remains open whether one also gets a sharper estimate in Theorem 1 , if $U$ is separable but $M$ (and thus $S$ ) is not.

Observe that we always have $\omega(M) \leq \omega_{1}(M)$. Hence, Theorem 1 might still provide a slightly better estimate for $\chi_{X}(M)$ than Theorem 2 (if $U$ has a nice geometry and $M$ is separable). However, if one is only interested in the question whether $M$ is precompact, the theorems are equivalent: 
Lemma 1. Let $X=L_{1}(S, U)$ with a Banach space $U$, and $M \subseteq X$. Then we have

$$
\omega(M) \leq \omega_{1}(M) \leq 2 \omega(M) .
$$

Proof. For any finite system of pairwise disjoint sets $E_{1}, \ldots, E_{n}$ of positive finite measure and any $x, y \in X$ we have

$$
\begin{aligned}
\| \sum_{k=1}^{n} & \left(\frac{1}{\operatorname{mes} E_{k}} \int_{E_{k}} x(s) d s\right) \chi_{E_{k}}-\sum_{k=1}^{n}\left(\frac{1}{\operatorname{mes} E_{k}} \int_{E_{k}} y(s) d s\right) \dot{\chi}_{E_{k}} \| \\
\quad & \sum_{k=1}^{n}\left|\int_{E_{k}} x(s) d s-\int_{E_{k}} y(s) d s\right| \\
& \leq \sum_{k=1}^{n} \int_{E_{k}}|x(s)-y(s)| d s \\
& \leq\|x-y\| .
\end{aligned}
$$

Hence, two applications of the triangle inequality imply

$$
\begin{aligned}
\| x- & \sum_{k=1}^{n}\left(\frac{1}{\operatorname{mes} E_{k}} \int_{E_{k}} x(s) d s\right) \chi \dot{E}_{k} \| \\
& \leq\left\|y-\sum_{k=1}^{n}\left(\frac{1}{\operatorname{mes} E_{k}} \int_{E_{k}} y(s) d s\right) \chi E_{k}\right\|+2\|x-y\| .
\end{aligned}
$$

For any $C>\omega(M)$ we find pairwise disjoint sets $E_{1}, \ldots, E_{n}$ of positive finite measure such that

$$
\sup _{x \in M} \inf _{u_{1}, \ldots, u_{n} \in U}\left\|x-\sum_{k=1}^{n} u_{k} X E_{k}\right\|<C .
$$

In particular, for any $x \in M$ we find a function $y=\sum u_{k} \chi E_{k}$ with $\|x-y\| \leq C$. Now (14) implies

$$
\left\|x-\sum_{k=1}^{n}\left(\frac{1}{\operatorname{mes} E_{k}} \int_{E_{k}} x(s) d s\right) \chi E_{k}\right\| \leq 2\|x-y\| \leq 2 C
$$

and so $\omega_{1}(M) \leq 2 C$

From the proof we can also see the following stability property of $\omega$ and $\omega_{1}$ :

Lemma 2. Let $X$ be a preideal space of functions $x: S \rightarrow U$, and $M, M_{0} \subseteq X$. If there is some $\delta>0$ such that for each function $x \in M$ there exists a function $y \in M_{0}$ with $\|x-y\| \leq \delta$, then $\omega(M) \leq \omega\left(M_{0}\right)+\delta$. In the case $X=L_{1}(S, U)$, we also have $\omega_{1}(M) \leq \omega_{1}\left(M_{0}\right)+2 \delta$.

Proof. The statement for $\omega(M)$ is a straightforward application of the triangle inequality. Thus, let $X=L_{1}(S, U)$. For any $C>\omega_{1}\left(M_{0}\right)$, we find finitely many pairwise disjoint sets $E_{1}, \ldots, E_{n}$ of positive finite measure such that

$$
\left\|y-\sum_{k=1}^{n}\left(\frac{1}{\operatorname{mes} E_{k}} \int_{E_{k}} y(s) d s\right) \chi_{E_{k}}\right\| \leq C \quad\left(y \in M_{0}\right) .
$$


For any $x \in M$ we find some $y \in M$ with $\|x-y\| \leq \delta$. By (14) and the triangle inequality, we get

$$
\left\|x-\sum_{k=1}^{n}\left(\frac{1}{\operatorname{mes} E_{k}} \int_{E_{k}} x(s) d s\right) \chi E_{k}\right\| \leq C+2 \delta .
$$

Since $x \in M$ was arbitrary, this shows $\omega_{1}(M) \leq C+2 \delta$

Except for possibly the precompactness of $M(s)$, the conditions of Theorems 1 and 2 are even necessary for the precompactness of $M$ :

Proposition 1. Let $X$ be a regular preideal space of functions $x: S \rightarrow U$ with a Banach space $U$. Then we have

$$
\chi X(M) \geq \omega(M)
$$

and, in the case $X=L_{1}(S, U)$, also

$$
\chi X(M) \geq \frac{1}{2} \omega_{1}(M)
$$

Moreover,

$$
\chi x(M) \geq \inf _{m e s E<\infty} \sup _{x \in M}\left\|P_{E} \subset x\right\|
$$

and

$$
\chi x(M) \geq \limsup _{\delta \rightarrow 0} \sup _{\operatorname{mes} D \leq \delta} \sup _{x \in M}\left\|P_{D} x\right\| .
$$

In particular, if $M$ is precompact, then $M$ has equicontinuous norm and satisfies $\omega(M)$ $=0\left(\right.$ and $\omega_{1}(M)=0$ in the case $\left.X=L_{1}(S, U)\right)$.

Proof. Observe that (16) is a consequence of (15) and (13). Let us now prove the statement for the case that $M \subseteq X$ is a finite set.

That the right-hand side of (17) and (18) vanishes in this case follows immediately from the definition of regular spaces and the triangle inequality. To see that $\omega(M)=0$, let $\varepsilon>0$ be given and choose a set $E$ of finite measure such that $\left\|P_{E^{c}} x\right\|<\varepsilon$ for each $x \in M$. Since the support of $P_{E} x$ has finite measure, there is a sequence $y_{n}$ of simple functions with $y_{n}(s) \rightarrow P_{E} x(s)$ and $\left|y_{n}(s)\right| \leq\left|P_{E} x(s)\right|$ for almost all $s$ (see, e.g., [14: Lemma 4.1.1]). Since $X$ is regular, Lebesgue's dominated convergence theorem for regular preideal spaces [27: Theorem 3.3.6] implies $\left\|y_{n}-P_{E} x\right\| \rightarrow 0(n \rightarrow \infty)$. Thus, for any $\varepsilon>0$ and any $x \in M$, we find a simple function $y=\sum u_{k} \chi E_{k}$ with $\chi E_{k} \in X$ and $\left\|y-P_{E} x\right\| \leq \varepsilon$. By considering a common refinement, we may assume that the sets $E_{k}$ are pairwise disjoint and independent of $x$. ( $M$ is finite!). Since $\|x-y\|=\left\|\left(P_{E} x-y\right)+P_{E^{c}} x\right\| \leq 2 \varepsilon$, we have $\omega(M) \leq 2 \varepsilon$.

Now we attack the general case. Given $C>\chi x(M)$, we find a finite $C$-net $N \subseteq X$ for $M$. By what we had proved, we find for each $\varepsilon>0$ some set $E$ of finite measure, some $\delta>0$, and finitely many pairwise disjoint sets $E_{1}, \ldots, E_{n}$ with $\chi_{E_{k}} \in X$ such that for each $y \in N$ we have the estimates $\left\|P_{E c} y\right\| \leq \varepsilon,\left\|P_{D} y\right\| \leq \varepsilon$ (mes $D \leq \delta$ ), and

$$
\inf _{u_{1}, \ldots, u_{n} \in U}\left\|y-\sum_{k=1}^{n} u_{k} \chi E_{k}\right\| \leq \varepsilon .
$$


For each $x \in M$, we find some $y \in N$ with $\|x-y\| \leq C$. But now the triangle inequality implies $\left\|P_{E^{c}} x\right\| \leq C+\varepsilon,\left\|P_{D} x\right\| \leq C+\varepsilon(\operatorname{mes} D \leq \delta)$, and

$$
\inf _{u_{1}, \ldots, u_{n} \in U}\left\|x-\sum_{k=1}^{n} u_{k} \chi E_{k}\right\| \leq C+\varepsilon
$$

We may conclude that (17), (18) and (15) are satisfied

Even for $U=\mathbb{R}^{n}$ our compactness criteria are apparently new, and give even a precise formula for the Hausdorff measure of non-compactness for sets with equicontinuous norm (be aware that the apparently simpler formula in $L_{p}$ given in [1] is false; see also [22]):

Corollary 1. Let $X$ be a regular preideal space of functions $x: S \rightarrow U$ with a finite-dimensional space $U$. Then a set $M \subseteq X$ is precompact if and only if the following holds:

1. $M$ is bounded and has equicontinuous norm.

2. $\omega(M)=0$ or, if $X=L_{1}(S, U)$, equivalently $\omega_{1}(M)=0$.

Moroever, if $M$ is bounded and has equicontinuous norm and the measure space $S$ is separable, we have the identity

$$
\chi \chi \chi(M)=\omega(M) .
$$

Under the assumption that $M$ be precompact in measure, another formula for $\chi_{X}(M)$ was given in [29] (related results in $L_{p}$ can also be found in [28]).

Let us remark that the precompactness of $M(s)$ is far from being necessary for the precompactness of $M$. Let us give a typical class of counterexamples in $L_{p}([0,1], U)$ :

Example 6. Let $X=L_{p}([0,1], U)$ with $1 \leq p<\infty$. Given an arbitrary countable set $U_{0} \subseteq U$, we may define a precompact countable set $M \subseteq X$ such that $M(s) \equiv U_{0}$ for all $s$.

Indeed, without loss of generality let $0 \in U_{0}$ (otherwise we consider a translation of $U_{0}$ and add a constant function afterwards). Choose a sequence $u_{k} \in U_{0}$ such that $U_{0}=\left\{u_{1}, u_{2}, \ldots\right\}$ and $\left|u_{k}\right| \leq 2^{\frac{k}{2 p}}$ for all sufficiently large $k$ (repeat some elements, if necessary): Now define a sequence $x_{n}$ in the following way: For $n=2^{k}+j \quad(j=$

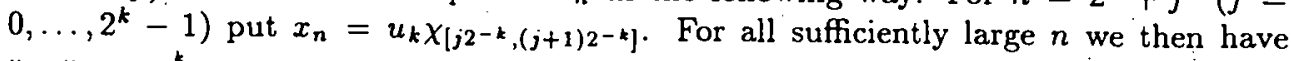
$\left\|x_{n}\right\| \leq 2^{-\frac{k}{2 p}} \rightarrow 0$, hence the set $M=\left\{x_{1}, x_{2}, \ldots\right\}$ is precompact. Moreover, $M(s)=U_{0}$ for each $s$.

However, in the theory of vector-valued integral and functional equations, one usually has an estimate for $\chi(M(s))$, and our results are applicable. However, $\omega(M)=0$ may be interpreted as some "equicontinuity" condition on the family $M$ of functions. Thus, it is not surprising that this condition may also be formulated similarly to the compactness criterion of Riesz, at least for locally compact groups: 
Theorem 3. Let $S$ be a locally compact Hausdorff group with a left Haar measure and unit element 0 . Let $X$ be a regular ideal space of locally integrable functions $x$ : $S \rightarrow U$ which contains all functions $\chi_{K}$ with compact $K \subseteq S$. Let $M \subseteq X$ satisfy

$$
\lim _{h \rightarrow 0} \sup _{x \in M} \sup _{t \in S}\left\|x^{h o t}-x^{t}\right\|=\lim _{h \rightarrow 0} \sup _{x \in M}\left\|x^{-h}-x\right\|=0
$$

where we have put $x^{h}(s)=x(h \circ s)$ and $x^{-h}(s)=x(s \circ h)$. Assume that either $\operatorname{supp} M$ exists and is contained in a compact set, or that $M$ has equicontinuous norm and even

$$
\lim _{\delta \rightarrow 0} \sup _{\operatorname{mes} D \leq \delta} \sup _{t \in S} \sup _{x \in M}\left\|P_{D} x^{\imath}\right\|=0 .
$$

Then $\omega(M)=0$.

Proof. Let us first assume that supp $M \subseteq K$ for some compact set $K$. Let $\varepsilon>0$ be given. Choose some compact neighborhood $H$ of 0 such that

$$
\left\|x^{-h}-x\right\|<\varepsilon \quad(x \in M, h \in H) .
$$

Observe that $0<$ mes $H<\infty$. Since all functions $x \in X$ are locally integrable, we may define the "inverse" convolutions ("Steklov functions")

$$
F x(t)=\frac{1}{\operatorname{mes} H} \int_{H} x(t \circ s) d s=\int_{S} x^{t}(s) y(s) d s
$$

where we have put $y(s)=(\operatorname{mes} H)^{-1} \chi_{H}$. Observe that the function $w(t, s)=x(t \circ s) y(s)$ vanishes for $s \notin H$ or for $t \notin H^{-1} \mathrm{o} K=: K_{0}$. Since $K_{0}$ and $H$ are compact and thus have finite measure, we may apply Fubini-Tonelli's theorem on the measure space $K_{0} \times H$ : The Borel function $v(t, s)=t o s$ has a (compact) range of finite measure and the property that preimages of null sets are null sets. Indeed, if $N \subseteq S$ is a null set, it is contained in a Borel null set $N_{0}$. Then $v^{-1}\left(N_{0}\right)$ is a Borel set, in particular measurable, and by Fubini-Tonelli we have

$$
\operatorname{mes}\left(v^{-1}(N)\right) \leq \int_{K_{0}} \int_{H} \chi_{v^{-1}\left(N_{0}\right)}(t, s) d s d t=\int_{K_{0}} \int_{H} \chi_{N_{0}}(t \circ s) d s d t=0 .
$$

We may conclude that $w$ is measurable on $K_{0} \times H$. Indeed, if $x_{n}$ are simple Borel functions on the range of $v$ which converge a.e. to $x$, then $x_{n} \circ v$ is measurable and converges a.e. to $x \circ v$. Hence, also the function $z(t, s)=(x(t \circ s)-x(t)) y(s)$ is measurable on $K_{0} \times H$. Since $X$ is regular, this implies by [27: Theorem 4.4.2] that the abstract function $Z: H \rightarrow X, Z(s)=\left(x^{-s}-x\right) y(s)$ is measurable. By [27: Theorem 4.4.3] the integral over this abstract function (the following formula shows that it exists) may be evaluated as the pointwise integral (a.e.), and thus we have the estimate

$$
\begin{aligned}
\|F x-x\| & =\left\|t \mapsto \int_{S}\left(x^{t}(s)-x(t)\right) y(s) d s\right\| \\
& =\left\|t \mapsto \int_{H}\left(x^{-s}(t)-x(t)\right) y(s) d s\right\| \\
& =\left\|\int_{H} Z(s) d s\right\| \leq \int_{H}\|Z(s)\| d s=\int_{S}\left\|x^{-s}-x\right\| y(s) d s \leq \varepsilon .
\end{aligned}
$$


It follows with Lemma 2 for the set $F M=\{F x: x \in M\}$ that $\omega(M) \leq \omega(F M)+\varepsilon$.

To estimate $\omega(F M)$, we argue as follows: Since all functions in $P_{H} X$ are integrable, the function $y$ must even belong to the associate space $\left(P_{H} X\right)^{\prime}$ (see, e.g., [27: Theorem 3.4.2]), i.e. we may apply Hölder's inequality for ideal spaces: For all $t, h \in S$, and all $x \in X$, we get the estimate

$$
|F x(h \circ t)-F x(t)|=\left|\int_{S}\left(x^{h \circ t}(s)-x^{t}(s)\right) y(s) d s\right| \leq\left\|x^{h \circ t}(s)-x^{t}\right\|\|y\|_{\left(P_{H} X\right)^{\prime}} .
$$

By assumption, we find an open neighborhood $O$ of 0 such that for all $h \in O$ the righthand side is less than $\frac{\varepsilon}{\left\|\chi_{0}\right\|}$ for each $x \in M$ and each $t \in S$. From what we have shown above, the support of $F M$ is contained in $K_{0}$. By the compactness there exist finitely many $t_{j} \in S$ such that $K_{0}$ is covered by the sets $O \circ t_{j}$. By considering a common refinement, we may divide $K_{0}$ into finitely many pairwise disjoint sets $E_{1}, \ldots, E_{m}$ such that each $E_{k}$ is contained in some set $O \circ t_{j(k)}$. Given $x \in M$, we put $u_{k}=F x\left(t_{j(k)}\right)$. Then for each $s \in E_{k}, s=h \circ t_{j(k)}$ with some $h \in O$, the estimate

$$
\left|F x(s)-u_{k}\right|=\left|F x\left(h \circ t_{j(k)}\right)-F x\left(t_{j(k)}\right)\right| \leq \frac{\varepsilon}{\left\|\chi K_{0}\right\|}
$$

holds. Summing up these inequalities, we find

$$
\left|F x(s)-\sum_{k=1}^{n} u_{k} \chi E_{k}(s)\right| \leq \varepsilon \frac{\chi K_{0}(s)}{\left\|\chi K_{0}\right\|} .
$$

Taking the norm in $X$ for the functions on both sides of this inequality, and observing that $E_{1}, \ldots, E_{m}$ was independent from $x \in M$, we may conclude that $\omega(F M) \leq \varepsilon$. Hence, $\omega(M) \leq 2 \varepsilon$.

Let us now assume that $M$ has equicontinuous norm and (20) holds. Given $\varepsilon>0$, we find a set $E$ of finite measure and $\delta>0$ such that $\left\|P_{E^{c}} x\right\| \leq \varepsilon$ and $\left\|P_{D} x\right\| \leq$ $\varepsilon(\operatorname{mes} D \leq \delta)$. Since $E$ is of finite measure and inner-regular, it contains a compact set $K \subseteq E$ with $\operatorname{mes}(E \backslash K) \leq \delta$. We may conclude that $\left\|P_{K^{c}} x\right\|=\left\|P_{E^{\mathrm{c}}} x+P_{E \backslash K} x\right\| \leq 2 \varepsilon$ for all $x \in M$. Hence, Lemma 2 implies $\omega_{X}(M) \leq \omega_{X}\left(P_{K} M\right)+2 \varepsilon$. Thus, if we can apply the statement for $P_{K} M$ in place of $M$, we are done.

To show that $P_{K} M$ satisfies (19), we first prove that the measure of the sets

$$
K_{t, h}=\left(t^{-1} \circ K\right) \Delta\left(t^{-1} \circ h^{-1} \circ K\right) \quad \text { and } \quad K_{h}=K \Delta\left(K \circ h^{-1}\right)
$$

tends to 0 as $h \rightarrow 0$, uniformly for $t \in S$. Indeed, given $\varepsilon>0$, there is a continuous function $z$ with compact support such that $\left\|z-\chi_{K}\right\|_{L_{1}}<\varepsilon$. Then we have $\left\|z^{h}-\chi_{K}^{h}\right\|_{L_{1}}<$ $\varepsilon$ and $\left\|z^{-h}-\chi_{K}^{-h}\right\|_{L_{1}}<\Delta(h) \varepsilon$, where $\Delta$ denotes the modular function of the Haar measure. We thus find

$$
\begin{aligned}
\operatorname{mes}\left(K_{t, h}\right) & =\left\|\chi_{K}^{t}-\chi_{K}^{h o t}\right\|_{L_{1}} \\
& =\left\|\chi K-\chi_{K}^{h}\right\|_{L_{1}} \\
& \leq\|\chi K-z\|_{L_{1}}+\left\|z-z^{h}\right\|_{L_{1}}+\left\|z^{h}-\chi_{K}^{h}\right\| \\
& \leq 2 \varepsilon+\left\|z-z^{h}\right\|_{L_{1}}
\end{aligned}
$$


and

$$
\begin{aligned}
\operatorname{mes}\left(K_{h}\right) & =\left\|\chi K-\chi_{K}^{-h}\right\|_{L_{1}} \\
& \leq\|\chi K-z\|_{L_{1}}+\left\|z-z^{-h}\right\|_{L_{1}}+\left\|z^{h}-\chi_{K}^{h}\right\|_{L_{1}} \\
& \leq(1+\Delta(h)) \varepsilon+\left\|z-z^{-h}\right\|_{L_{1}} .
\end{aligned}
$$

In view of the equicontinuity of $z$ and $\Delta(h) \rightarrow 1$ as $h \rightarrow 0$, we thus have $\sup _{t} \operatorname{mes}\left(K_{t, h}\right)$ $\rightarrow 0$ and $\operatorname{mes}\left(K_{h}\right) \rightarrow 0$, as claimed.

Hence, by (20), we find for each $\varepsilon>0$ some neighborhood $H$ of 0 such that for all $h \in H, t \in S, x \in X$ the inequalities $\left\|P_{K_{t, h}} x^{t}\right\|<\varepsilon$ and $\left\|P_{K_{h}} x\right\|<\varepsilon$ hold. In view of

$$
\begin{aligned}
\left|\left(P_{K} x\right)^{h \circ t}(s)-\left(P_{K} x\right)^{t}(s)\right| & =\left|\chi_{K}^{\text {hot }}(s)\left(x^{h \circ t}(s)-x^{t}(s)\right)-\left(\chi_{K}^{\text {hot }}(s)-\chi_{K}^{t}(s)\right) x^{t}(s)\right| \\
& \leq\left|x^{h \circ t}(s)-x^{t}(s)\right|+\left|P_{K, ~} x^{t}(s)\right|
\end{aligned}
$$

and

$$
\begin{aligned}
\left|\left(P_{K} x\right)^{-h}(s)-\left(P_{K} x\right)(s)\right| & =\left|\chi_{K}^{-h}(s)\left(x^{-h}(s)-x(s)\right)+\left(\chi_{K}^{-h}(s)-\chi_{K}(s)\right) x(s)\right| \\
& \leq\left|x^{-h}(s)-x(s)\right|+\left|P_{K_{\mathrm{h}}} x(s)\right|
\end{aligned}
$$

we thus find that

$$
\limsup _{h \rightarrow 0} \sup _{x \in M} \sup _{t \in S}\left\|\left(P_{K} x\right)^{h \circ t}-\left(P_{K} x\right)^{h}\right\| \leq \varepsilon
$$

and

$$
\limsup _{h \rightarrow 0} \sup _{x \in M}\left\|\left(P_{K} x\right)^{-h}-P_{K} x\right\| \leq \varepsilon .
$$

Hence, the set $P_{K} M$ satisfies (19), as claimed

If the ideal space $X$ is even invariant under left-translations of $S$ in the sense that $x \in X$ implies $x^{t} \in X$ and $\|x\|=\left\|x^{t}\right\|$ (like, e.g., $X=L_{p}(S, U)$ ), then condition (19) may of course equivalently be replaced by

$$
\lim _{h \rightarrow 0} \sup _{x \in M}\left\|x^{h}-x\right\|=\lim _{h \rightarrow 0} \sup _{x \in M}\left\|x^{-h}-x\right\|=0 .
$$

Moreover, in this case condition (20) may be dropped, since it already is a consequence of the fact that $M$ has equicontinuous norm.

Corollary 2. Let $X=L_{p}\left(\mathbb{R}^{n}, U\right) \quad(1 \leq p<\infty)$ with a Banach space $U$. If $U$ has the retraction property, we put $L=1$, otherwise $L=2$. Let $M \subseteq X$ have equicontinuous norm and satisfy

$$
\lim _{h \rightarrow 0} \sup _{x \in M} \int_{S}|x(s+h)-x(s)|^{p} d s=0 .
$$

Then for each $y \in X$ which satisfies $y(s) \geq \chi\left(M_{0}(s)\right)$ a.e. for each countable $M_{0} \subseteq M$, we have

$$
\chi x(M) \leq 2 L\|y\| \text {. }
$$

Moreover, the factor 2 may be dropped if either $M$ is separable and $U$ has the exhaustion property, or if $p=1$ and either $M$ or $U$ are separable. If $U$ has the retraction property, 
the family $\mathcal{M}=\left\{\chi\left(M_{0}(\cdot)\right): M_{0} \subseteq M\right.$ countable $\}$ consists of measurable functions, and thus we may choose $y=\sup \mathcal{M}$.

The measurability of the function $\chi\left(M_{0}(\cdot)\right)$ has been proved in [4] (see also [12, 19]). Here, $\sup \mathcal{M}$ denotes the smallest upper bound with respect to the order "almost everywhere"; this supremum exists (and is measurable) by a theorem of Kantorovich, if the underlying measure space is $\sigma$-finite (see, e.g., [30]).

A special version of Corollary 2 for $L_{1}([a, b], U)$ with a separable Banach space $U$ and countable $M$ has been proved in [20] (observe that Corollary 2 shows that it is not necessary for that result that $M$ be countable; moreover, our boundedness assumptions are much weaker). An analogous version of the mentioned result from [20] for the Kuratowski measure of non-compactness can be found in [25]. Applications of these special versions to Volterra equations in Banach spaces can be found in the earlier mentioned papers $[20,21,23,26]$.

\section{Proofs of Theorems 1 and 2}

The heart of the proofs is the following result which has implicitly been shown in [18: Propostion 1.4] (see also [19: Proposition 2]).

Lemma 3. Let $U$ be a normed space, and $U_{1} \subseteq U_{2} \subseteq \ldots$ be finite-dimensional subspaces. Then for any countable bounded $M \subseteq U, M=\left\{u_{1}, u_{2}, \ldots\right\}$, we have the estimate

$$
\chi U(M) \leq \lim _{k \rightarrow \infty} \limsup _{n \rightarrow \infty} \operatorname{dist}\left(u_{n}, U_{k}\right) .
$$

Moreover, if $U$ is separable, then there exists a sequence of finite-dimensional subspaces $U_{1} \subseteq U_{2} \subseteq \ldots$ with $U=\overline{U U_{k}}$; and for any such sequence we have the equality

$$
\chi U(M)=\lim _{k \rightarrow \infty} \limsup _{n \rightarrow \infty} \operatorname{dist}\left(u_{n}, U_{k}\right) .
$$

Proof. Let $C$ be larger than the right-hand side of (23). Then we find some $k$ and $n_{0}$ with the following property: For any $n \geq \dot{n}_{0}$ there is some $v_{n} \in U_{k}$ with $\left\|u_{n}-v_{n}\right\| \leq C$. Since the set of all $v_{n}$ is a bounded subset of the finite-dimensional space $U_{k}$, we find for any $\varepsilon>0$ a finite $\varepsilon$-net $N \subseteq U_{k}$ for this set. In particular, for any $n \geq n_{0}$ we find some $u \in N$ with $\left\|u_{n}-u\right\| \leq C+\varepsilon$. Hence, $N \cup\left\{u_{1}, \ldots, u_{n_{0}}\right\}$ is a finite $(C+\varepsilon)$-net for $M$, and so $\chi U(M) \leq C+\varepsilon$. This proves (23).

If $U$ is separable, there exists a countable dense subset $\left\{w_{1}, w_{2}, \ldots,\right\}$, and one may choose $U_{k}=\operatorname{span}\left\{w_{1}, \ldots, w_{k}\right\}$. To see (24), let $C>\chi_{U}(M)$ and $N$ be a finite $C$-net for $M$. Given $\varepsilon>0$, we find some $k$ with $\operatorname{dist}\left(u, U_{k}\right) \leq \varepsilon$ for each $u \in N$. This implies $\operatorname{dist}\left(u_{n}, U_{k}\right) \leq C+\varepsilon$ for each $n$, and it follows that the right-hand side of (24) is bounded by $C+\varepsilon$, hence bounded by $\chi \cup(M)$

The crucial point in Lemma 3 is that it allows to calculate $\chi$ from the "outside", i.e. without explicit knowledge of a finite $\varepsilon$-net. To get an estimate if $U$ does not have a nice geometry, we need the following fact. 
Lemma 4. Each separable Banach space $U_{0}$ is isometrically and linear embedded into a separable Banach space $V$ with the $(1,2)$-exhaustion property.

Proof. By [5: Chapter XI, Theorem $9(\S 8)], U_{0}$ can be mapped isometrically and linear onto a closed linear subspace of the space $V=C([0,1])$. Since $V$ has a monotone base (namely the classical Schauder system; see, e.g., [24: Chapter I, §3] or [14: Chapter 1]), Example 3 implies that $V$ has the $(1,2)$-exhaustion property

We emphasize that the proof of Lemma 4 does not require the (uncountable) axiom of choice: The Hahn-Banach extension theorem (which is invoked in the cited result) can be proved "constructively" in separable spaces (see [10]).

We do not know whether any separable Banach space may be embedded (isometrically) into a separable Banach space with the $\left(p_{0}, q_{0}\right)$-property with $q_{0}<2$. If this is the case, one may strengthen Theorem 1 . More precisely, if $C([0,1])$ may be embedded into a separable Banach space with the $\left(p_{0}, q_{0}\right)$-exhaustion property, then our proof shows that we may replace (5) and (6) by the estimates

$$
\chi_{i}(M) \leq 2\left(p_{0} \omega(M)+q_{0}\|y\|\right) \quad \text { and } \quad \alpha(M) \leq 2\left(p_{0} \omega(M)+q_{0}\|y\|\right)
$$

respectively. However, it is not very reasonable that better constants than in Lemma 4 are possible: As a matter of fact, the "universal" space $U=C([0,1])$ has the $(p, q)$ exhaustion property only for $p \geq 1$ and $q \geq 2$. The latter follows from the result in [6] which states that any compact operator $K$ in $U$ satisfies $\|I+K\|=1+\|K\|$ : Let $U_{0}=U$, and $P_{k}: V=U \rightarrow U_{k}$ be as in Definition 2. Since $P_{k}$ is compact, we have $\left\|I-P_{k}\right\|=1+\left\|P_{k}\right\| \geq 2$. Thus, for any $\varepsilon>0$ we find some $u \in U_{0}=V, u \neq 0$, such that $\left|u-P_{k} u\right| \geq(2-\varepsilon)|u| \geq(2-\varepsilon) \operatorname{dist}\left(u, U_{k}\right)$. With the notation of Definition 2, this means $q_{k} \geq 2$ for each $k$, and so $q \geq 2$, as claimed.

Proof of Theorem 1. Let us first reduce the statement to the case that the functions in $M$ are uniformly dominated by a function $\rho_{\chi} E \in X$, where $E$ has finite measure:

Suppose that $M \subseteq X$ has equicontinuous norm. Given $\varepsilon>0$, let $E_{0}$ be a set of finite measure with

$$
\sup _{x \in M}\left\|P_{E_{0}^{c}} x\right\|<\varepsilon
$$

and $\delta>0$ be such that

$$
\sup _{\operatorname{mes} D \leq \delta} \sup _{x \in M}\left\|P_{D} x\right\|<\varepsilon .
$$

Since $E_{0}$ has finite measure, $S_{0}=\operatorname{supp} P_{E_{0}} X$ exists [27: Theorem 2.2.4], and by [27: Theorem 2.2.5] there is a set $E \subseteq S_{0}$ with mes $\left(S_{0} \backslash E\right)<\delta$ such that $\chi E \in X$. For all $x \in X$ we have $P_{E^{c} x}=P_{E_{0}^{c}} x+P_{S_{0} \backslash E} x$, and so

$$
\left\|P_{E^{\mathrm{c}} \mathrm{x}}\right\| \leq 2 \varepsilon .
$$

Note that $P_{E} M$ is bounded in $X$. By [27: Corollary 3.1.3], the set $P_{E} M$ thus is bounded in measure. In particular, we find some natural number $n_{0}$ such that the measure of the set $\left\{s:\left|P_{E} x(s)\right|>n_{0}\right\}$ is less than $\delta$ for each $x \in M$. Then also the measure of the 
set $D(x)=\left\{s \in E: x(s) \notin K_{n_{0}}\right\}$ is less than $\delta$ for each $x \in M$. Now we consider the set

$$
M_{\varepsilon}=\left\{P_{E \backslash D(x)} x: x \in M\right\} .
$$

For each $x \in M$ the corresponding function $z=P_{E \backslash D(x)} x \in M_{\varepsilon}$ satisfies

$$
\|x-z\|=\left\|P_{E^{\mathrm{e}} x}+P_{D(x)} x\right\| \leq 3 \varepsilon .
$$

Since each measure $\gamma \in\left\{\chi, \alpha, \chi_{i}\right\}$ is continuous with respect to the Hausdorff distance (see, e.g., [1]), we find for each $\varepsilon_{0}>0$ some $\varepsilon>0$ such that the sizes $\gamma(M)$ and $\gamma\left(M_{\varepsilon}\right)$ differ by at most $\varepsilon_{0}$. By Lemma 2 , the sizes $\omega\left(M_{\varepsilon}\right)$ and $\omega(M)$ differ by at most $\varepsilon$. Moreover, the corresponding estimate (3) respectively (4) holds for $M_{e}$ in place of $M$ by our construction. Thus, it suffices to prove the statement for $M_{e}$ in place of $M$ (for all sufficiently small $\varepsilon>0$ ). But the set $M_{\varepsilon}$ has by construction the additional required property (observe that $K_{n_{0}}$ is bounded by some number $\rho>0$ ).

Thus, we assume without loss of generality that all functions in $M$ are uniformly dominated by $\rho \chi E \in X$ with mes $E<\infty$ (and hence take their values in some $K_{n_{0}}$ ).

First, assume additionally that $M$ is countable. Since the support of each function in $M$ has finite measure, the functions are essentially separably valued (see [9: Section III.6/Theorem 10]). This means that after modifying the (countably many!) functions on a null set, we may assume that they have separable range. For any $C>\omega(M)$ we can find a finite partition $E_{1}, \ldots, E_{m}$ of $E$ (with measurable sets $E_{i}$ ) with the following property: For each $x \in M$ there is some function $z=z_{x}$ of the form

$$
z=\sum_{i=1}^{m} u_{i} \times E_{i}
$$

with $u_{i} \in U$ such that $\|x-z\|<C$. Let $H$ denote the closed linear hull of all values of the functions in $M$ and all values of the functions $z_{x}$. Then $H$ is separable.

If $U$ has the $L$-retraction property, we choose $R$ corresponding to Definition 1 (for the subspace $H$ ), and denote the closed linear hull of the range of $R$ by $U_{0}$; otherwise, we put $U_{0}=H$ and $R=I$. In both cases, we have

$$
\chi U_{0}(M(s)) \leq y(s) \quad \text { a.e. }
$$

Indeed, if $U$ has the $L$-retraction property, and $N \subseteq U$ is a finite $\varepsilon$-net for $M(s)$, then $R(N) \subseteq U_{0}$ is a finite $L \varepsilon$-net for $R(M(s))=M(s)$; hence $\chi U_{0}(M(s)) \leq L_{\chi}(M(s)) \leq$ $y(s)$. In the other case, our assumptions imply $\chi U_{0}(M(s)) \leq \chi_{i}(M(s)) \leq y(s)$.

In order to prove (8), choose $P_{n}, V, U_{n}, p$, and $q$ corresponding to Definition 2. If we want to prove (6) instead, we choose $V$ as in Lemma 4 , and then $P_{n}$ and $U_{n}$ as in Definition 2 (corresponding to $U_{0}:=V$ ); in this case we put $p=1, q=2$, and tacitly identify $U_{0}$ with a subspace of $V$ in the following.

To clarify notation, we denote by $X_{V}$ the preideal space of functions $x: S \rightarrow V$ which has the same real form than $X$. Let $X_{k}$ denote the finite-dimensional subspace of all functions of the form (25) with $u_{i} \in U_{k}$. We claim that for each $\varepsilon>0$ there is some $K$ such that

$$
\operatorname{dist}_{X V}\left(x, X_{k}\right) \leq p C+q\left\|\operatorname{dist}_{V}\left(x(\cdot), U_{k}\right)\right\|+\varepsilon \quad(x \in M, k \geq K) .
$$


Indeed, we have

$$
\begin{aligned}
\left|P_{k} z_{x}(s)-x(s)\right| & =\left|P_{k}\left(z_{x}(s)-x(s)\right)+\left(P_{k} x(s)-x(s)\right)\right| \\
& \leq\left\|P_{k}\right\|\left|z_{x}(s)-x(s)\right|+q_{k} \operatorname{dist}_{v}\left(x(s), U_{k}\right)
\end{aligned}
$$

which implies that

$$
\left\|P_{k} z_{x}-x\right\| \leq\left\|P_{k}\right\|\left\|z_{x}-x\right\|+q_{k}\left\|\operatorname{dist}_{V}\left(x(\cdot), U_{k}\right)\right\| .
$$

Since $P_{k} z_{x} \in X_{k}$, this proves (26).

For definiteness, let $M=\left\{x_{1}, x_{2}, \ldots\right\}$. Putting $x_{n, k}(s)=\sup _{m \geq n} \operatorname{dist} v\left(x_{m}(s), U_{k}\right)$, we have by (26) that

$$
\sup _{m \geq n} \operatorname{dist}_{X_{v}}\left(x_{m}, X_{k}\right) \leq q\left\|x_{n, k}\right\|+p C+\varepsilon .
$$

Since $\left|x_{n, k}(s)\right| \leq \rho \chi E(s)$ and

$$
x_{n, k}(s) \rightarrow y_{k}(s)=\underset{n \rightarrow \infty}{\limsup } \operatorname{dist} v\left(x_{n}(s), U_{k}\right)
$$

we may pass to the limit $n \rightarrow \infty$ in (27) by Lebesgue's dominated convergence theorem for preideal spaces (see [27: Theorem 3.3.6]). Thus, we get

$$
\underset{n \rightarrow \infty}{\limsup } \operatorname{dist}_{X v}\left(x_{n}, X_{k}\right) \leq q\left\|y_{k}\right\|+p C+\varepsilon
$$

Observe that (24) implies

$$
y_{k}(s) \rightarrow \lim _{k \rightarrow \infty} \limsup _{n \rightarrow \infty} \operatorname{dist}_{V}\left(x_{n}(s), U_{k}\right)=\chi V(M(s)) \leq \chi U_{0}(M(s)) \leq y(s) .
$$

Hence, passing to the limit $k \rightarrow \infty$ in (28), we find

$$
\lim _{k \rightarrow \infty} \lim _{n \rightarrow \infty} \operatorname{dist}_{X_{V}}\left(x_{n}, X_{k}\right) \leq q\|y\|+p C+\varepsilon
$$

Now (23) implies $\chi_{X_{V}}(M) \leq q\|y\|+p C+\varepsilon$. Letting $\varepsilon \rightarrow 0$, we thus find $\chi_{X_{V}}(M) \leq$ $p C+q\|y\|$. Now the proof of (8) is completed, since $V \subseteq U$ implies $X_{V} \subseteq X$, and so $\chi x(M) \leq \chi x_{v}(M)$. For the proof of (6), we observe that $\alpha(M) \leq 2 \chi_{X_{v}}(\bar{M})$ and that $\alpha(M)$ is the same in the space $X$ as in the space $X_{V}$.

Now we consider the case that $M$ is not necessarily countable: If $M$ is separable in $X$, there is a countable subset $M_{0} \subseteq M$ with $\overline{M_{0}}=M$ (see, e.g., [2: Lemma 2.6]). Then we have $\gamma(M)=\gamma\left(M_{0}\right)$ for $\gamma \in\left\{\alpha, \chi \chi, \chi_{i}\right\}$. Since evidently $\omega\left(M_{0}\right) \leq \omega(\dot{M})$, we get estimate (6) respectively (8) by applying the corresponding estimate for the countable set $M_{0}$.

In general; there exists a countable $M_{0} \subseteq M$ such that $\chi_{i}(M) \leq \alpha\left(M_{0}\right) \leq 2 \chi x\left(M_{0}\right)$ (see, e.g., [4]). Applying estimate (6) respectively (8) for $M_{0}$ in place of $M$, we get the desired estimate (5) rspectively (7) 
The proof of Theorem 2 is based on the following result.

Theorem 4. Let $X=L_{1}(S, U)$, and let $M \subseteq X$ be uniformly dominated by an integrable function. Let $y$ be integrable such that

$$
\chi_{i}\left(M_{0}(s)\right) \leq y(s) \text { a.e. } \quad\left(M_{0} \subseteq M \text { countable }\right)
$$

If $M$ is countable, (29) needs to hold only for $M_{0}=M$. If $U$ has the $L$-retraction property, we may replace (29) by

$$
L_{\chi}\left(M_{0}(s)\right) \leq y(s) \text { a.e. } \quad\left(M_{0} \subseteq M \text { countable }\right)
$$

Then the estimate

$$
\chi_{i}\left(\left\{\int_{S} x(s) d s: x \in M\right\}\right) \leq 2 \int_{S} y(s) d s
$$

holds. Moreover, if either $M$ is countable or $U$ is separable, we even have

$$
\chi\left(\left\{\int_{S} x(s) d s: x \in M\right\}\right) \leq \int_{S} y(s) d s .
$$

Theorem 4 is proved in [4]. Actually, the result in [4] is only formulated for the case that $L=1$ and that estimate (29) respectively (30) holds everywhere, but an inspection of the proof shows that the result also holds for $L>1$ and that the exceptional null set may depend on $M_{0}$. Also, the proof of Theorem 4 is based on Lemma 3. It is worth noting that the idea to use Lemma 3 to prove results like Theorem 4 is apparently due to Mönch [18, 19] (although the proof of [19: Proposition 3] contained a small mistake in the application of Fatou's lemma which however can be avoided by using Lebesgue's theorem instead). We remark that in [12] it is shown by means of an example that, if $U$ does not have the retraction property, one may not replace (29) by (30) with $L<2$ (even for $S=[0,1], U=l_{\infty}$ and countable $M \subseteq C(S, U)$ ).

Let us note that the condition that $M \subseteq X=L_{1}(S, U)$ be uniformly dominated by an integrable function in Theorem 4 can actually be replaced by the weaker condition that all functions in $M$ have $\sigma$-finite support and

$$
\lim _{n \rightarrow \infty} \sup _{x \in M} \int_{D_{n}}|x(s)| d s=0
$$

for each sequence of measurable sets $D_{n} \downarrow \emptyset$. Indeed, under the additional assumption that $M$ is a bounded subset of $X=L_{1}(S, U)$, this has been proved in [4]. The general case may be established by modifying the proof in [4] by isolating certain atoms of the measure space. However, we shall not apply this more general result (although this would allow to prove a slight generalization of Theorem 2).

Proof of Theorem 2. With the same argument as in the proof of Theorem 1, we can reduce the statement to the case that all functions in $M$ are uniformly dominated by a function $\rho \chi_{E} \in X$. Thus, let us assume this. 
For any $C>\omega_{1}(M)$ we find finitely many pairwise disjoint sets $E_{1}, \ldots, E_{n}$ of positive finite measure such that

$$
\sup _{x \in M}\left\|x-\sum_{k=1}^{n}\left(\frac{1}{\operatorname{mes} E_{k}} \int_{E_{k}} x(s) d s\right) \chi E_{k}\right\|<C .
$$

By Theorem 4, the Hausdorff measure of non-compactness (in $U$ ) of the set

$$
V_{k}=\left\{\int_{E_{k}} x(s) d s: x \in M\right\} \subseteq U
$$

is bounded by

$$
c_{k}:=2 \int_{E_{k}} y(s) d s
$$

(and we may even drop the factor 2 , if $U$ is separable or $M$ is countable). This estimate means that, given $\varepsilon>0^{\circ}$, we find a finite $\left(c_{k}+\varepsilon\right)$-net $N_{k} \subseteq U$ for $V_{k}$. Now put

$$
N=\left\{\sum_{k=1}^{n} \frac{1}{\operatorname{mes} E_{k}} u_{k} \chi E_{k}: u_{k} \in N_{k}\right\} \text {. }
$$

For each $x \in M$, there exist $u_{k} \in N_{k}$ such that

$$
\left|\int_{E_{k}} x(s) d s-u_{k}\right| \leq c_{\dot{k}}+\varepsilon \quad(k=1, \ldots, n) .
$$

The function $z=\sum\left(\text { mes } E_{k}\right)^{-1} u_{k} \chi E_{k} \in N$ thus satisfies

$$
\begin{aligned}
\| \sum_{k=1}^{n} & \left(\frac{1}{\operatorname{mes} E_{k}} \int_{E_{k}} x(s) d s \chi E_{k}\right)-z \| \\
& \backslash \sum_{k=1}^{n}\left|\int_{E_{k}} x(s) d s-u_{k}\right| \leq \sum_{k=1}^{n}\left(c_{k}+\varepsilon\right) \leq 2 \int_{S} y(s) d s+n \varepsilon=2\|y\|+n \varepsilon .
\end{aligned}
$$

By (33) and the triangle inequality; this implies $\|x-z\| \leq C+2\|y\|+n \varepsilon$. Hence, $N \subseteq X$ is a finite $(C+2\|y\|+n \varepsilon)$-net for $M$, i.e. $\chi x(M) \leq C+2\|y\|+n \varepsilon$. Now first letting $\varepsilon \rightarrow 0$ ( $n$ depends on $C$ !) and then $C \rightarrow \omega_{1}(M)$, we find (11). If $U$ is separable or $M$ is countable, we may drop the factor 2 in all above formulas.

The case that $M$ is uncountable but separable in $X$, is exceptional. To get the better estimate (12) in this case, we choose a countable dense $M_{0} \subseteq M$ (recall [2: Lemma 2.6]). Then $\chi x(M)=\chi x\left(M_{0}\right)$, and by what we have proved so far, $\chi x\left(M_{0}\right) \leq$ $\omega_{1}\left(M_{0}\right)+\|y\| \leq \omega_{1}(M)+\|y\|$

Let us remark that Theorems 1 and 2 hold slightly more general:

1. Instead of requiring that $M$ have equicontinuous norm, it suffices to require that each countable subset of $M$ have equicontinuous norm. 
2. Actually, it is not necessary to assume that the sets $K_{n}$ are bounded.

The generalization of Statement 1 is of interest, e.g., for $X=L_{p}(S, U)$, if $S$ is not $\sigma$-finite. For example, (32) is equivalent to the statement that $M$ has equicontinuous norm in $L_{1}(S, U)$, provided that $M$ has $\sigma$-finite support [27: Lemma 3.3.4]. But the latter is true for countable sets $M \subseteq L_{1}(S, U)$ while not necessarily for the whole set $M$.

The generalization of Statement 2 is only interesting in view of the fact that $\chi_{i}$ is not monotone, in general.

Let us briefly sketch, how the proof of Theorem 1 has to be modified to cover these cases. For Statement 1 it suffices to change the order of argumentation: One first has to reduce the statement to countable $M$ (with the same arguments as in the proof). Then the reduction to the case that all functions in $M$ be uniformly dominated by some $\rho \chi E$ requires only the equicontinuity of the bounded set $M$.

The changes for Statement 2 are more difficult to describe. Actually, our proof shows for the case that $M$ is countable and all functions are uniformly dominated by some $\rho \chi E$ slightly more than as is claimed in the statement. Namely, it is not required that $\chi_{i}\left(M(s) \cap K_{n}\right) \leq y(s)$ but it suffices that $\chi_{H}(M(s)) \leq y(s)$ for some separable subspace $H \subseteq U$ which contains (essentially) all values of the functions of $M$. Observing that $\chi_{H}$ is monotone, the other parts of the proof actually reduce the theorem to this special case, if we replace $D(x)$ by $D(x)=\left\{s:|x(s)|>n_{0}\right\}$ (put $\rho=n_{0}$ ).

It would lead too far to describe the necessary changes in the proof of Theorem 2 here in detail. Theorem 4 has to be modified appropriately (in particular, one has to introduce the sets $K_{n}$ already in the statement of Theorem 4).

Let us finally note that Theorem 1 also holds (in principle) for the case that $X$ is only quasinormed, i.e. instead of the triangle inequality of the norm, we only have

$$
\|x+y\| \leq c(\|x\|+\|y\|)
$$

with some constant $c<\infty$; the most prominent example is $X=L_{p}(S, U) \quad(0<p<1)$. In this case, one has to replace inequalities (5) - (8) by the respective estimates

$$
\begin{aligned}
\chi_{i}(M) & \leq 2 c^{4}(c \omega(M)+2\|y\|) \\
\alpha(M) & \leq 2 c^{5}(c \omega(M)+2\|y\|) \\
\chi_{i}(M) & \leq 2 c^{4}(c p \omega(M)+q\|y\|) \\
\chi_{X}(M) & \leq c^{4}(\operatorname{cp} \omega(M)+q\|y\|) .
\end{aligned}
$$

Moreover, for countable $M$ one may divide the right-hand sides of (34) and (35) by $c$.

Of course, one may discuss whether it makes sense at all to consider the Hausdorff and Kuratowski measures of non-compactness in non-metric spaces. 


\section{References}

[1] Akhmerov, R. R. at al.: Measures of Noncompactness and Condensing Operators. Basel - Boston - Berlin: Birkhäuser Verlag 1992.

[2] Alt, H. W.: Lineare Funktionalanalysis, 2nd ed. Berlin - Heidelberg - New York: SpringerVerlag 1992.

[3] Amir, D. and J. Lindenstrauss: The structure of weakly compact sets in Banach spaces. Ann. Math. (2) 88 (1968), $34-46$.

[4] Appell, J., Väth, M., and A. Vignoli: Compactness and existence results for ordinary differential equations in Banach spaces. Z. Anal. Anw. 18 (1999)3 (to appear).

[5] Banach, S.: Introduction to Linear Operations. Amsterdam - New York - Oxford: NorthHolland 1987; translation of: Théorie des Opérations Linéaires. Warsaw: Polish Sci. Publ. 1979.

[6] Daugavet, I. K.: On a property of completely continuous operators in the space $C$ (in Russian). Uspekhi Mat. Nauk 18 (1963)5, $157-158$.

[7] Day, M. M.: Normed linear spaces, 3rd ed. Berlin - Heidelberg - New York: SpringerVerlag 1973.

[8] Diestel, J.: Geometry of Banach Spaces - Selected Topics. Lect. Notes Math. 485 (1975), $1-282$.

[9] Dunford, N. and J. T. Schwartz: Linear Operators. Vol. I: General Theory, 3rd ed. New York: Int. Publ. 1966.

[10] Garnir, H. G., de Wilde, M., and J. Schmets: Analyse fonctionnelle. Théorie constructive des espaces linéaires à semi-normes. Tome I: Théorie générale. Basel - Stuttgart: Birkhäuser Verlag 1968.

[11] Gribanov, Yu. I.: Two compactness criteria for sets in Banach function spaces (in Russian). Kazan. Gos. Univ. Uchen. Zap. 125 (1966)2, 32 - 39.

[12] Heinz, H.-P.: On the behaviour of measures of noncompactness with respect to differentiation and integration of vector-valued functions. Nonlin. Anal. 7 (1983), $1351-1371$.

[13] Krasnoselskiǐ, M. A. et al.: Integral Operators in Spaces of Summable Functions (in Russian). Moscow: Nauka 1958; Engl. transl.: Leyden: Noordhoff 1976.

[14] Lindenstrauss, J. and L. Tzafriri: Classical Banach Spaces. Vol. I: Sequence spaces. Berlin - Heidelberg - New York: Springer-Verlag 1977.

[15] Luxemburg, W. A. J.: Notes on Banach function spaces, Part XVI $A$. Proc. Netherl. Acad. Sci. (A) 68 (1965), 646 - 657; Indag. Math. 27 (1965), 646 - 657.

[16] Luxemburg, W. A. J.: Notes on Banach function spaces, Part XVI $\mathrm{I}_{\text {. }}$. Proc. Netherl. Acad. Sci. (A) 68 (1965), 658 - 667; Indag. Math. 27 (1965), 658 - 667.

[17] Luxemburg, W. A. J. and A. C. Zaanen: Notes on Banach function spaces, Part XII. Proc. Netherl. Acad. Sci. (A) 67 (1964), 519 - 529; Indag. Math. 26 (1964), 519 - 529.

[18] Mönch, H.: Boundary value problems for nonlinear ordinary differential equations in Banach spaces. Nonlinear Anal. 4 (1980), 985 - 999.

[19] Mönch, H. and G.-F. von Harten: On the Cauchy problem for ordinary differential equations in Banach spaces. Arch. Math. (Basel) 39 (1982), $153-160$.

[20] Orlicz, W. and S. Szufla: On some classes of nonlinear Volterra integral equations in Banach spaces. Bull. Acad. Polon. Sci. Sér. Sci. Math. 30 (1982), 239 - 250.

[21] Orlicz, W.: On the structure of $L_{\Phi}$ solution sets of integral equations in Banach spaces. Studia Math. 77 (1984), $465-477$. 
[22] Otáhal, A.: Measure of noncompactness of subsets of Lebesgue spaces. Cas. Pestovani. Mat. 103 (1978), 67 - 72.

[23] Pluciennik, R. and S. Szufla: Nonlinear Volterra integral equations in Orlicz spaces. Demonstratio Math. 17 (1984), 515 - 532.

[24] Singer, I.: Bases in Banach spaces, Vol. I. Berlin - Heidelberg - New York: Springer-Verlag 1970.

[25] Szufla, S.: Appendix to the paper "An existence theorem for the Urysohn integral equation in Banach spaces". Comment. Math. Univ. Carolinae 25 (1984), $763-764$.

[26] Szufla, S.: On the Aronszajn property for integral equations in Banach space. Atti Accad. Naz. Lincei Rend. Cl. Sci. Fis. Mat. Natur. (8) 83 (1989), 93 - 99.

[27] Väth, M.: Ideal Spaces. Lect. Notes Math. 1664 (1997), 1 - 146.

[28] Yerzakova, N. A.: On the Hausdorff internal measure of noncompactness (in Russian). In: Operator Theory in Functional Spaces (ed.: E. M. Semenov). Interuniv. Collect. of Sci. Works. Voronezh: Izd. Univ. 1983, pp. $37-45$.

[29] Yerzakova, N. A.: On measures of non-compactness in regular spaces. Z. Anal. Anw. 15 (1996), 299 - 307.

[30] Zaanen, A. C.: Integration. Amsterdam: North-Holland Publ. Comp. 1967.

[31] Zabreǐko, P. P.: Ideal spaces of functions, Part I (in Russian). Vestnik Jaroslav. Univ. 8 (1974), 12 - 52.

Received 28.09.1998 